\title{
On the Differences Between Discretized and Continuous Stochastic Systems as Demonstrated by Learning Automata*
}

\author{
B. John Oommen ${ }^{\dagger}$ \\ School of Computer Science, Carleton University \\ Ottawa, Canada. K1S 5B6. \\ E-mail: oommen@scs.carleton.ca
}

\begin{abstract}
Stochastic Learning Automata (LA) are probabilistic finite state machines which have been used to model how biological systems can learn. The structure of such a machine can be fixed, or it can be changing with time. A LA can also be implemented by using action probability updating rules which may or may not depend on estimates from the Environment being investigated. During the initial years of research in the field of LA, these updating rules worked with the continuous probability space. In this paper, we will describe how LA can also be designed by discretizing the probability space. The paper ${ }^{1}$ will describe the design and analysis of both continuous and discretized LA, and will highlight the subtle differences between the corresponding learning machines, their convergence properties, and their learning capabilities.
\end{abstract}

Keywords: Learning Automata, Continuous Learning Automata, Discretized Learning Automata, Estimator-based Learning Automata

\section{Introduction}

\section{Learning Automata}

The goal of a Learning Automaton (LA) is to determine the optimal action out of a set of allowable actions, where the optimal action is defined as the action that maximizes the probability of being rewarded.

The functionality of the LA can be described in terms of a sequence of repetitive feedback cycles in which the automaton interacts with the Environment. During a

*The author dedicates this paper to Prof. Thathachar, his first mentor and teacher in the field of LA. Thank you, Professor Thathachar. I owe you much more than you realize!

${ }^{\dagger}$ Chancellor's Professor; Fellow: IEEE and Fellow: $I A P R$. The author also holds an Adjunct Professorship with the Department of Information and Communication Technology, University of Agder, Grooseveien 36, N-4876 Grimstad, Norway. This work was supported in part by the Natural Sciences and Engineering Research Council of Canada.

${ }^{1}$ The contents of this paper were presented as a Plenary/Keynote talk for the SSS'08 Conference in Kyoto in November 2008. This event was the $40^{\text {th }}$ conference in this series. cycle, the Automaton chooses an action, which triggers a response from the Environment, a response that can be either a reward or a penalty. The Automaton uses this response and the knowledge acquired in the past actions to determine which is the next action. By learning to choose the optimal action, the automaton adapts itself to the Environment. Excellent references that survey the field are the books by Lakshmivarahan [3], Narendra and Thathachar [7], Najim and Poznyak [6], and a recent special issue of the journal, IEEE Transactions on Systems, Man, and Cybernetics, Part B [8].

The learning paradigm as modeled by LA has found applications in systems that posses incomplete knowledge about the Environment in which they operate $[3,6,7,8]$. A variety of applications ${ }^{2}$ that use LA have been reported in the literature. They have been used in game playing, pattern recognition, object partitioning, parameter optimization and multi-objective analysis, telephony routing, priority assignments in a queuing system. They have also been used in statistical decision making, distribution approximation, natural language processing, modeling biological learning systems, string taxonomy, graph partitioning, distributed scheduling, network protocols (including conflict avoidance) for LANs, photonic LANs, star networks and broadcast communication systems, dynamic channel allocation, tuning PID controllers, assigning capacities in prioritized networks, map learning, digital filter design, controlling client/server systems, adaptive signal processing, vehicle path control, and even the control of power systems and vehicle suspension systems.

The beauty of incorporating LA in any particular application domain is, indeed, the elegance of the technology. Essentially, LA are utilized exactly as one would expect - by interacting with the "Environment" - which is the system from which the LA learns. For example, in parameter optimization the LA chooses a parameter, observes the effect of the parameter in the control

${ }^{2}$ The applications listed here are few, and the actual bibliographic citations are omitted due to space limitations. Indeed, this relatively new field has been "exploding". It has recently been enhanced by a spectrum of applications in computer science and engineering - from areas as diverse as the design of data structures, to the implementation of automatic navigation methods. 
loop, and then updates the parameter to optimize the objective function, where this updating is essentially achieved by the LA's stochastic updating rule. The details of how this is achieved in the various application domains involves modeling the "actions", and transforming the system's outputs so that they are perceived to be of a reward or penalty flavor. This is where the ingenuity of the researcher comes into the picture - this is often a thought-provoking task. In the first LA designs, the transition and the output functions were time invariant, and for this reason these LA were considered "fixed structure" automata [7, 8]. Tsetlin, Krylov, and Krinsky presented notable examples of this type of automata. Later, Vorontsova and Varshavskii introduced a class of stochastic automata known in literature as Variable Structure Stochastic Automata (VSSA). In the definition of a VSSA, the LA is completely defined by a set of actions (one of which is the output of the automaton), a set of inputs (one of which is usually the response of the Environment) and a learning algorithm, T. Within the context of this paper, the VSSA $[3,6,7,8]$ operates on a vector, called the Action Probability vector, $\mathbf{P}(t)$, where:

$\mathbf{P}(t)=\left[p_{1}(t), \ldots, p_{r}(t)\right]^{T}$,

where $p_{i}(t)(i=1, \ldots, r)$ is the probability that the automaton will select the action $\alpha_{i}$ at the time $t$ :

$p_{i}(t)=\operatorname{Pr}\left[\alpha(t)=\alpha_{i}\right], i=1, \ldots, r$, and it satisfies, $\sum_{i=1}^{r} p_{i}(t)=1$ for all ' $t$ '.

Note that the algorithm $T:[0,1]^{r} \times A \times B \rightarrow[0,1]^{r}$ is an updating scheme where $A=\left\{\alpha_{1}, \alpha_{2}, \ldots, \alpha_{r}\right\}, 2 \leq r<$ $\infty$, is the set of output actions of the automaton, and $B$ is the set of responses from the Environment. Thus, the updating is such that

$$
\mathbf{P}(t+1)=T(\mathbf{P}(t), \alpha(t), \beta(t)),
$$

where $\beta(t)$ is the response that the LA receives from the Environment.

If the mapping $T$ is chosen in such a manner that the Markov process has absorbing states, the algorithm is referred to as an absorbing algorithm. Families of VSSA that posses absorbing barriers have been studied in the literature $[3,6,7]$. Ergodic VSSA have also been reported and extensively studied $[3,6,7]$. These VSSA converge in distribution and thus, the asymptotic distribution of the action probability vector has a value that is independent of the corresponding initial vector. Thus, while ergodic VSSA are suitable for non-stationary environments, automata with absorbing barriers are preferred in stationary environments.

During the initial years of research in the field of LA, these updating rules worked with the continuous probability space. In practice, the relatively slow rate of convergence of these algorithms constituted a limiting factor in their applicability. In order to increase their speed of convergence, the concept of discretizing the probability space was first introduced in [13] and later extensively studied in $[1,2,4,5,9,10,11]$. This concept is implemented by restricting the probability of choosing an action to a finite number of values in the interval $[0,1]$. Following the discretization concept many of the continuous VSSA have been discretized. This paper describes, in a concise manner, how LA can be designed by discretizing the probability space. The rest of the paper will explain the design and analysis of various families of discretized LA, and will highlight the subtle differences between the corresponding learning machines, their convergence properties, and their learning capabilities.

\section{Discretized Learning Automata}

In the implementation of LA, the machine uses a Random-Number Generator (RGN) in determining which action to choose. In theory, an action probability can take any value between 0 and 1 , so the RNG is required to be very accurate. In practice, though, the probabilities are rounded-off to an accuracy depending on the architecture of the machine used to implement it.

In order to increase the speed of convergence of these algorithms and to minimize the requirements of the RNG, the probability space can be discretizied $[1,2,4,5,9,10,11,13]$. Analogous to the continuous algorithms, the discretized VSSA can be defined using probability updating functions, but these functions can take values in the discretized finite space. These values divide the continuous $[0,1]$ interval into a finite number of continuous subintervals. The Discrete LA are said to be linear if these subintervals have equal length, otherwise they are called nonlinear.

Like the continuous LA, the discretized LA can be analyzed using the theory of Markov chains, and can be divided into two categories: ergodic or absorbing. Various discrete automata have been presented in literature $[9,10,11,13]$. Indeed, all of the linear VSSA reported in the literature have corresponding discretized versions: i.e. the Discretized Linear Reward-Penalty automaton $\left(\mathrm{DL}_{\mathrm{RP}}\right)$, the Discretized Linear RewardInaction automaton $\left(\mathrm{DL}_{\mathrm{RI}}\right)$, and the Discretized Linear Inaction-Penalty automaton (DL $\left.\mathrm{DP}_{\mathrm{P}}\right)$ [9, 10, 11, 13] The paper mentions the similarities and dissimilarities between some continuous automata and their discrete counterparts.

\subsection{Discretized Linear Reward-Inaction LA}

The Discretized Linear Reward-Inaction (DL $\mathrm{RI}_{\mathrm{I}}$ ) automaton was the first discretized automaton presented in the literature [13]. In the following description of this automaton, only two actions are considered, but the same concept applies to a $r$-action $(2<r<\infty)$ automaton. The basic idea of the learning algorithm is to make discrete changes in the action probabilities. The probability space $[0,1]$ is divided into $N$ intervals, where $N$ is a resolution parameter and is recommended to be 
an even integer. Since it is a reward-inaction automaton, the updating equations do not modify the action probability vector when the Environment penalizes the automaton. When the response from the Environment is a reward, the automaton increases the probability of the action that has been chosen and decreases the probability for all the remaining actions.

The discretized automaton has a state associated with every possible probability value, which determines the following set of states: $Q=\left\{q_{1}, q_{2}, \cdots, q_{N}\right\}$. In any state $q_{i}$, the probability that the automaton chooses action $\alpha_{1}$ is $\frac{i}{N}$ and the probability to choose action $\alpha_{2}$ is $\left(1-\frac{i}{N}\right)$. The state transition map is defined by the following equations:

$$
\begin{aligned}
& q(t+1)=q_{i+1}, \quad \text { if } \alpha(t)=\alpha_{1} \text { and } \beta(t)=0 \\
& q(t+1)=q_{i-1}, \quad \text { if } \alpha(t)=\alpha_{2} \text { and } \beta(t)=0 \\
& q(t+1)=q_{i}, \quad \text { if } \alpha(t)=\alpha_{1} \text { or } \alpha_{2} \text { and } \beta(t)=1
\end{aligned}
$$

where $q(t)=q_{i} \neq q_{0}$ or $q_{N}$. It can be seen that both $q_{0}$ and $q_{N}$ are absorbing states. Based on the probabilities associated with each state, the automaton can be described entirely by the following action probability updating equations:

$$
\begin{array}{ll}
p_{1}(t+1)=\min \left\{1, p_{1}(t)+\frac{1}{N}\right\}, & \text { if } \alpha(t)=\alpha_{1}, \beta(t)=0 \\
p_{1}(t+1)=\max \left\{0, p_{1}(t)-\frac{1}{N}\right\}, & \text { if } \alpha(t)=\alpha_{2}, \beta(t)=0 \\
p_{1}(t+1)=p_{1}(t) & \text { if } \beta(t)=1 \\
p_{2}(t+1)=1-p_{1}(t+1) . &
\end{array}
$$

The algorithm starts with the initial action probability vector $\mathrm{P}(0)=\left[\frac{1}{2}, \frac{1}{2}\right]^{\mathrm{T}}$.

These equations indicate that $\{\mathbf{P}(t)\}$ behaves like a homogenous Markov chain with two absorbing states: $[1,0]^{\mathrm{T}}$ and $[0,1]^{\mathrm{T}}$. It possesses the following property.

Theorem I: The $\mathrm{DL}_{\mathrm{RI}}$ automaton is $\varepsilon$-optimal in all random Environments

Proof: The proof of the result is given in [10].

The difference between this algorithm and its continuous version is in the rate of convergence. Oommen and Hansen have performed simulations of the $\mathrm{L}_{\mathrm{RI}}$ and $\mathrm{DL}_{\mathrm{RI}}$ automata, and in all scenarios, the $\mathrm{DL}_{\mathrm{RI}}$ automaton is superior to the $\mathrm{L}_{\mathrm{RI}}$ automaton [10]. For example, their studies indicate that when the two automata were made to learn the best action in an Environment with $c_{1}=0.2$ and $c_{2}=0.6$, in 240 iterations the $\mathrm{L}_{\mathrm{RI}}$ automaton gave only an expected value of 0.99982 . The $\mathrm{DL}_{\mathrm{RI}}$ scheme gave an expected value of 0.99999 and subsequently the value stayed at unity. If a stopping criterion was used, it was seen that the $\mathrm{DL}_{\mathrm{RI}}$ automaton reached 0.99 accuracy in 125 iterations, and the $L_{R I}$ automaton reached the same accuracy in 135 iterations.

In [11], Oommen compared $\mathrm{DL}_{\mathrm{RI}}$ with some deter-

\begin{tabular}{|c|c|c|c|c|c|c|}
\hline$c_{1}$ & $c_{2}$ & $\mathrm{~N}$ & $E\left[p_{1}(\infty)\right]$ & $\begin{array}{l}\mathrm{DL}_{\mathrm{RI}} \\
\text { MTC }\end{array}$ & $\begin{array}{l}\text { Tsetlin } \\
p_{1}(\infty)\end{array}$ & $\begin{array}{c}\text { Krinsky } \\
p_{1}(\infty)\end{array}$ \\
\hline \multirow{3}{*}{0.4} & \multirow{3}{*}{0.8} & 4 & 0.84 & 6 & 0.80 & 0.80 \\
\hline & & 10 & 0.95 & 16 & 0.95 & 0.97 \\
\hline & & 20 & 0.99 & 31 & 0.99 & 0.99 \\
\hline \multirow{3}{*}{0.6} & \multirow{3}{*}{0.8} & 4 & 0.74 & 9 & 0.64 & 0.64 \\
\hline & & 10 & 0.86 & 26 & 0.72 & 0.81 \\
\hline & & 20 & 0.94 & 53 & 0.75 & 0.95 \\
\hline \multirow{3}{*}{0.7} & \multirow{3}{*}{0.8} & 4 & 0.65 & 11 & 0.57 & 0.57 \\
\hline & & 10 & 0.73 & 34 & 0.60 & 0.66 \\
\hline & & 20 & 0.81 & 76 & 0.60 & 0.79 \\
\hline
\end{tabular}
ministic automata. The Table 1 shows a comparison between the performance of various learning automata.

From these results, Oommen concluded that for Environments with $c_{1}>0.5$, the $\mathrm{DL}_{\mathrm{RI}}$ is more accurate
Table 1: Experimental comparative performance of DL $_{R I}$ with other FSSA [11]. The MTC is the Mean Time of Convergence of the LA.

than the Tsetlin automaton. Furthermore, for a fixed $\mathrm{N}$, as the difference between the penalty probabilities is decreased, the $\mathrm{DL}_{\mathrm{RI}}$ becomes more accurate than the Tsetlin and Krinsky automata. In all these Environments, Oommen observed that the $\mathrm{DL}_{\mathrm{RI}}$ automaton is much faster than the Tsetlin and Krinsky automata.

\subsection{Discretized Linear Inaction-Penalty LA}

Following the same method of discretization used for the reward-inaction algorithm, a discretized version of the Linear Inaction-Penalty algorithm, denoted as $\mathrm{DL}_{\mathrm{IP}}$, was developed [11]. The updating rules for the $\mathrm{DL}_{\mathrm{IP}}$ algorithm are defined in the following equations:

$$
\begin{array}{ll}
p_{1}(t+1)=\min \left\{1, p_{1}(t)+\frac{1}{N}\right\}, & \text { if } \alpha(t)=\alpha_{2}, \beta(t)=1 \\
p_{1}(t+1)=\max \left\{0, p_{1}(t)-\frac{1}{N}\right\}, & \text { if } \alpha(t)=\alpha_{1}, \beta(t)=1 \\
p_{1}(t+1)=p_{1}(t) \quad \text { if } \alpha(t)=\alpha_{1} \text { or } \alpha_{2}, \beta(t)=0 \\
p_{2}(t+1)=1-p_{1}(t) .
\end{array}
$$

The scheme possesses the following property.

Theorem II: The $\mathrm{DL}_{\mathrm{IP}}$ automaton is ergodic and expedient in all random Environments.

Proof: The proof of the result is given in [11].

Oommen also created an absorbing version of the $\mathrm{DL}_{\mathrm{IP}}$ automaton, denoted as $\mathrm{ADL}_{\mathrm{IP}}$. The idea was very simple: Whenever the LA reached one of its end states associated with the probabilities ' 0 ' or ' 1 ', the machine was artificially absorbed into this state. This absorbing version of the $\mathrm{DL}_{\mathrm{IP}}$ automaton is known as the Absorbing Discrete Linear Inaction-Penalty LA, and denoted by $\mathrm{ADL}_{\mathrm{IP}}$. It has the following property.

Theorem III: The ADL $\mathrm{IP}$, obtained by rendering the end states of the $\mathrm{DL}_{\mathrm{IP}}$ to be artificially absorbing, is $\varepsilon$-optimal in all random Environments.

Proof: The result is included in the paper by Oommen [11].

The $\mathrm{ADL}_{\mathrm{IP}}$ is the first and only inaction-penalty algorithm proved to be $\varepsilon$-optimal [11]. Although it is $\varepsilon$ optimal, simulation results have shown that this scheme is very accurate but slow in convergence [11]. When 
the penalty probabilities are high, the automaton utilizes many more responses of the Environment than a reward-inaction automaton.

\subsection{Discretized Linear Reward-Penalty LA}

The Discretized Linear Reward-Penalty (DLRP) automaton, as its continuous version, reacts to both reward and penalty responses of the Environment. Similar to the $\mathrm{DL}_{\mathrm{RI}}$ and the $\mathrm{DL}_{\mathrm{IP}}$ automata, $\mathrm{DL}_{\mathrm{RP}}$ updates its action probabilities in steps of size $1 / N$, where $N$ is the resolution parameter. The updating rules for this automaton are given by the following equations:

$$
\begin{aligned}
& \left.p_{1}(t+1)=\min \left\{1, p_{1}(t)+\frac{1}{N}\right\}\right\} \begin{array}{r}
\text { if } \alpha(t)=\alpha_{1}, \beta(t)=0 \\
\text { or } \alpha(t)=\alpha_{2}, \beta(t)=1
\end{array} \\
& \left.p_{1}(t+1)=\max \left\{0, p_{1}(t)-\frac{1}{N}\right\}\right\} \begin{aligned}
\text { if } \alpha(t)=\alpha_{1}, \beta(t)=1 \\
\text { or } \alpha(t)=\alpha_{2}, \beta(t)=0
\end{aligned} \\
& p_{2}(t+1)=1-p_{1}(t+1) .
\end{aligned}
$$

The $\mathrm{DL}_{\mathrm{RP}}$ possesses the following property.

Theorem IV: The DL $\mathrm{RP}_{\mathrm{RP}}$ automaton is ergodic and $\varepsilon$-optimal in all random Environments whenever $c_{\text {min }}<$ 0.5 .

Proof: The proof of the result is due to Oommen and Christensen and given in [9].

Oommen and Christensen also showed that by making a stochastic modification to the transition function, the automata can be made ergodic. This was done by treating every Penalty response from the Environment as a simulated Penalty with probability 0.5 , and as a simulated Reward with probability 0.5 . This modified version of the $\mathrm{DL}_{\mathrm{RP}}$ automaton is known as the Modified Discrete Linear Reward-Penalty LA, (MDL $\mathrm{RP}_{\mathrm{RP}}$, and has the following property.

Theorem V: The MDL ${ }_{\mathrm{RP}}$, modified by treating every Penalty response from the Environment as a simulated Penalty with probability 0.5 , and as a simulated Reward with probability 0.5 , is ergodic and $\varepsilon$-optimal in all random Environments.

Proof: The proof of the result, due to Oommen and Christensen, is found in [9].

The $\mathrm{MDL}_{\mathrm{RP}}$ is the only known ergodic linear rewardpenalty scheme, which is $\varepsilon$-optimal in all random Environments.

Oommen and Christensen also created an absorbing version of the $\mathrm{DL}_{\mathrm{RP}}$ automaton denoted as $A D L_{R P}$. The idea followed the exact same principles of rendering the $\mathrm{DL}_{\mathrm{IP}}$ absorbing. Whenever the LA reached one of its end states associated with the probabilities ' 0 ' or ' 1 ', the machine was artificially absorbed into this state. This absorbing version of the $\mathrm{DL}_{\mathrm{RP}}$ automaton is known as the Absorbing Discrete Linear RewardPenalty $L A,\left(\mathrm{ADL}_{\mathrm{RP}}\right)$, and has the following property.

Theorem VI: The $\mathrm{ADL}_{\mathrm{RP}}$, obtained by rendering the end states of the $\mathrm{DL}_{\mathrm{RP}}$ to be artificially absorbing, is $\varepsilon$-optimal in all random Environments.

Proof: The proof of the result is found in [9].

The $\mathrm{ADL}_{\mathrm{RP}}$ is the only symmetric $\varepsilon$-optimal learning automata known. Simulation results indicated that the $\mathrm{ADL}_{\mathrm{RP}}$ scheme is extremely accurate and fast in convergence [9]. Oommen and Christensen have also made a comparative study of the performance and accuracy of some of the discrete linear automata [9]. The results of this study, presented in Table 2, show that the ADL $R$ P scheme is superior based on the criteria of both speed and accuracy.

These results indicate, for example, that if $N=$ $10, c_{1}=0.6$ and $c_{2}=0.8$, the $\mathrm{DL}_{\mathrm{RI}}$ scheme converges with an expected accuracy of 0.855 , and the MTC was 25.58 iterations. With the same parameters, the $\mathrm{ADL}_{\mathrm{IP}}$ scheme converged with a greater accuracy (0.93) but the MTC was much bigger, 499.11 iterations. The results for the $\mathrm{ADL}_{\mathrm{RP}}$ have shown that it converged with an accuracy of 0.93 and the MTC was 32.45 iterations.

Although, in this section, we presented only linear schemes, it is important to note that discrete nonlinear schemes have also been developed [11]. A description of these is omitted here in the interest of brevity. The next section presents a new category of learning algorithms, the estimator algorithms.

\section{Continuous Estimator Algorithms}

\subsection{Overview}

In the quest to design faster converging learning algorithms, Thathachar and Sastry have opened another path by introducing a new class of algorithms, called estimator algorithms $[12,14]$. The main feature of these algorithms is that they maintain running estimates for the penalty probability of each possible action and use them in the probability updating equations. The purpose of these estimates is to crystallize the confidence in the reward capabilities of each action. In their characteristics, these algorithms model the behavior of a person that is trying to choose an action in a random Environment. In this task, the most common and simple approach is to try each action a number of times and to estimate the probability of reward for each action. The person will most likely choose the action that has the highest reward estimate; however, unlike straightforward estimation, the superior actions are also chosen in a more likely manner in the estimation process.

From this perspective, all the algorithms presented in the previous sections are non-estimator algorithms. The main difference between the estimator algorithms and the non-estimator algorithms lies in the way the action probability vector is updated. The non-estimator algorithms update the probability vector based directly on the response of the Environment. If the chosen action is rewarded, then the automaton increases the probability of choosing this action at the next time instant. Otherwise, the action probability of the selected 
Table 2: Comparative performance of the $\mathrm{DL}_{\mathrm{RI}}, \mathrm{ADL}_{\mathrm{IP}}$, and $\mathrm{ADL}_{\mathrm{RP}} \mathrm{LA}$. In all these cases, $c_{2}=0.8$.

\begin{tabular}{|c|c|c|c|c|c|c|c|}
\hline \multirow{2}{*}{$N$} & $c_{1}$ & \multicolumn{2}{|c|}{$\mathrm{DL}_{\mathrm{RI}}$} & \multicolumn{2}{|c|}{ ADL } & \multicolumn{2}{c|}{ ADL } \\
\cline { 3 - 8 } & & $E\left[p_{1}(\infty)\right]$ & MTC & $E\left[p_{1}(\infty)\right]$ & MTC & $E\left[p_{1}(\infty)\right]$ & MTC \\
\hline \multirow{4}{*}{4} & 0.2 & 0.896 & 4.61 & 0.960 & 9.87 & 0.95 & 2.965 \\
& 0.4 & 0.843 & 6.04 & 0.825 & 10.65 & 0.86 & 3.730 \\
& 0.6 & 0.741 & 8.52 & 0.655 & 10.57 & 0.72 & 4.830 \\
\hline \multirow{3}{*}{10} & 0.2 & 0.980 & 11.46 & 1.00 & 70.28 & 1.00 & 8.220 \\
& 0.4 & 0.951 & 16.15 & 1.00 & 196.78 & 0.98 & 14.88 \\
& 0.6 & 0.855 & 25.58 & 0.93 & 499.11 & 0.93 & 32.45 \\
\hline
\end{tabular}

action could be decreased.

The estimator algorithms are characterized by the use of the estimates for each action. The change of the probability of choosing an action is based on its current estimated mean reward, and possibly on the feedback of the Environment. The Environment determines the probability vector indirectly, through the calculation of the reward estimates for each action. Even when the chosen action is rewarded, there is a possibility that the probability of choosing another action is increased.

For the definition of an estimator learning automaton, a vector of reward estimates $\hat{\mathbf{D}}(t)$ must be introduced. Hence $[12,14]$, the state vector $\mathrm{Q}(t)$ is defined as $\mathbf{Q}(t)=<\mathbf{P}(t), \hat{\mathbf{D}}(t)>$ where $\hat{\mathbf{D}}(t)=\left[\hat{d}_{1}(t), \cdots, \hat{d}_{r}(t)\right]$.

Thathachar and Sastry have shown that the estimator algorithms exhibit a superior speed of convergence when compared with the non-estimator algorithms $[12,14]$. In 1989, Oommen and Lanctôt introduced discretized versions of the estimator algorithms, and have shown that the discretized estimator algorithms are even faster than their continuous counterparts [5].

The next sub-sections describe the class of continuous estimator algorithms.

\subsection{Pursuit Algorithm}

Thathachar and Sastry $[12,14]$ introduced the concept of estimator algorithms by presenting a Pursuit Algorithm that implemented a reward-penalty learning philosophy, denoted by $\mathrm{CP}_{\mathrm{RP}}$. As its name reveals, this algorithm is characterized by the fact that it pursues the action that is currently estimated to be the optimal action. The algorithm achieves this by increasing the probability of the current optimal action if the chosen action was either rewarded or penalized by the Environment.

The $\mathrm{CP}_{\mathrm{RP}}$ algorithm involves three steps $[12,14]$. The first step consists of choosing an action $\alpha(t)$ based on the probability distribution $\mathbf{P}(t)$. Whether the automaton is rewarded or penalized, the second step is to increase the component of $\mathrm{P}(t)$ whose reward estimate is maximal (the current optimal action), and to decrease the probability of all the other actions. The probability of the current optimal action is increased with a value directly proportional to the distance to unity, namely $1-p_{m}(t)$. All the other probabilities are decreased proportional to the distance to zero, i.e. $p_{i}(t)$. The last step is to update the running estimates for the probability of being rewarded. For calculating the estimates, two more vectors are introduced: $W(t)$ and $\mathrm{Z}(t)$, where $Z_{i}(t)$ is the number of times the $i^{\text {th }}$ action has been chosen, and $W_{i}(t)$ is the number of times the action $\alpha_{i}$ has been rewarded. Then, the estimate vector $\hat{\mathbf{D}}(t)$ can be calculated using the following formula:

$$
\hat{d}_{i}(t)=\frac{W_{i}(t)}{Z_{i}(t)} \text { for } i=1,2, \cdots, r .
$$

The $\mathrm{CP}_{\mathrm{RP}}$ algorithm is similar in design to the $\mathrm{L}_{\mathrm{RP}}$ algorithm, in the sense that both algorithms modify the action probability vector $\mathbf{P}(t)$ if the response from the Environment is a reward or a penalty. The difference occurs in the way they approach the solution. The $L_{R P}$ algorithm moves $\mathbf{P}(t)$ in the direction of the most recently rewarded action or in the direction of all the actions not penalized, whereas the $\mathrm{CP}_{\mathrm{RP}}$ algorithm moves $\mathbf{P}(t)$ in the direction of the action which has the highest reward estimate. Thathachar and Sastry proved that this algorithm is $\varepsilon$-optimal in every stationary Environment. Also, comparing the performance of the $\mathrm{CP}_{\mathrm{RP}}$ and $\mathrm{L}_{\mathrm{RI}}$ automata, the authors have shown that the $\mathrm{CP}_{\mathrm{RP}}$ algorithm converges up to seven times faster than the $L_{R I}$ automaton $[12,14]$. We formally present the $\mathrm{CP}_{\mathrm{RP}}$ algorithm below. 


\section{ALGORITHM CP $\mathrm{RP}$ \\ Parameters}

- $\lambda$ : the speed of learning parameter, where $0<\lambda<1$

- $m$ : index of the maximal component of $\hat{\mathbf{D}}(t), \hat{d}_{m}(t)=$ $\max _{i=1,2, \ldots, r}\left\{\hat{d}_{i}(t)\right\}$

- $W_{i}(t)$ : the number of times the $i^{t h}$ action has been rewarded up to the time $t$, with $1 \leq i \leq r$

- $Z_{i}(t)$ : the number of times the $i^{\text {th }}$ action has been chosen up to the time $t$, with $1 \leq i \leq r$

Method

\section{Initialization}

$p_{i}(t)=1 / r$, for $1 \leq i \leq r$

Initialize $\hat{\mathbf{D}}(t)$ by picking each action a small number of times.

Repeat

Step 1: At time $t$ pick $\alpha(t)$ according to probability distribution $\mathbf{P}(t)$, Let $\alpha(t)=\alpha_{i}$.

Step 2: If $\alpha_{m}$ is the current optimal action and $j \neq m$, update $\mathbf{P}(t)$ according to the following equations:

$$
\begin{aligned}
p_{m}(t+1) & =p_{m}(t)+\lambda\left(1-p_{m}(t)\right) \\
p_{j}(t+1) & =p_{j}(t)-\lambda p_{j}(t)
\end{aligned}
$$

Step 3: Update $\hat{\mathbf{D}}(t)$ according to the following:

$$
\left.\begin{array}{rl}
W_{i}(t+1) & =W_{i}(t)+(1-\beta(t)) \\
Z_{i}(t+1) & =Z_{i}(t)+1 \\
\hat{d}_{i}(t+1) & =\frac{W_{i}(t+1)}{Z_{i}(t+1)} \\
W_{j}(t+1) & =W_{j}(t) \\
Z_{j}(t+1) & =Z_{j}(t) \\
\hat{d}_{j}(t+1) & =\hat{d}_{j}(t)
\end{array}\right\} \text { for all } j \neq i
$$

\section{End Repeat}

\section{END ALGORITHM CP $R$}

\subsection{The TSE Algorithm}

Thathachar and Sastry in $[12,14]$ introduced a more sophisticated estimator algorithm, which we refer to as the TSE Algorithm. Being an estimator algorithm, it considers the reward estimates in calculating the action probability vector. The algorithm increases the probabilities for all the actions that have a higher estimate than the estimate of the chosen action, and decreases the probabilities of all the actions with a smaller estimate. The probabilities are updated based on both the reward estimates $\hat{\mathrm{D}}(t)$ and the action probability vector $\mathbf{P}(t)$, as shown below in the formal algorithm.

\section{ALGORITHM TSE \\ Parameters}

- $\lambda$ : the speed of learning parameter, where $0<\lambda<1$

- $m$ : index of the maximal component of $\hat{\mathbf{D}}(t), \hat{d}_{m}(t)=$ $\max _{i=1,2, \ldots, r}\left\{\hat{d}_{i}(t)\right\}$

- $W_{i}(t)$ : the number of times the $i^{\text {th }}$ action has been rewarded up to the time $t$, with $1 \leq i \leq r$

- $Z_{i}(t)$ : the number of times the $i^{\text {th }}$ action has been chosen up to the time $t$, with $1 \leq i \leq r$

- $S_{i j}(t)$ : an indicator function

$$
S_{i j}(t)= \begin{cases}0 & \text { if } \hat{d}_{i}(t) \leq \hat{d}_{j}(t) \\ 1 & \hat{d}_{i}(t)>\hat{d}_{j}(t)\end{cases}
$$

- $f: f:[-1,1] \mapsto[-1,1]$ a monotonic, increasing function satisfying $f(0)=0$.

\section{Method}

\section{Initialization}

$p_{i}(t)=1 / r$, for $1 \leq i \leq r$;

Initialize $\hat{\mathbf{D}}(t)$ by picking each action a small number of times

\section{Repeat}

Step 1: At time $t$ pick $\alpha(t)$ according to probability distribution $\mathbf{P}(t)$; Let $\alpha(t)=\alpha_{i}$;

Step 2: Update $\mathrm{P}(t)$ according to the following equations:

$$
\begin{aligned}
& p_{j}(t+1)=p_{j}(t)-\lambda\left[f\left(\hat{d}_{i}(t)-\hat{d}_{j}(t)\right)\right] \times \mathcal{S}, j \neq i \\
& p_{i}(t+1)=p_{i}(t)+\lambda \sum_{j \neq i}\left[f\left(\hat{d}_{i}(t)-\hat{d}_{j}(t)\right)\right] \times \mathcal{S}
\end{aligned}
$$

where $\left.\mathcal{S}=\left[S_{i j}(t) p_{j}(t)+S_{j i}(t)\right] \frac{p_{i}(t)}{r-1}\left(1-p_{j}(t)\right)\right]$

Step 3: Update $\hat{\mathbf{D}}(t)$ as done in Algorithm $\mathrm{CP}_{\mathrm{RP}}$

End Repeat

\section{END ALGORITHM TSE}

It is important to notice that $\mathbf{P}(t)$ depends indirectly on the response of the Environment. The feedback from the Environment changes the values of the reward estimate vector, which affects the values of the functions $f$ and $S_{i j}$.

The detailed description of the algorithm indicates that if the $i^{\text {th }}$ action is rewarded, all the probabilities $p_{j}(t)$ that correspond to actions with reward estimates higher than the reward estimate $\hat{d}_{i}(t)$ are updated using the following equation:

$$
p_{j}(t)=p_{j}(t)-\lambda \cdot f\left(\hat{d}_{i}(t)-\hat{d}_{j}(t)\right) \cdot \frac{p_{i}(t)}{r-1} \cdot\left(1-p_{j}(t)\right)
$$

Since $\hat{d}_{i}(t)<\hat{d}_{j}(t)$, the sign of the function $f\left(\hat{d}_{i}(t)-\right.$ $\left.\hat{d}_{j}(t)\right)$ is negative, and thus, the probability $p_{j}(t)$ increases proportional to $\left(1-p_{j}(t)\right)$.

For all the actions with reward estimates smaller than $\hat{d}_{i}(t)$, the probabilities are updated based on the following equation:

$$
p_{j}(t+1)=p_{j}(t)-\lambda \cdot f\left(\hat{d}_{i}(t)-\hat{d}_{j}(t)\right) \cdot p_{j}(t)
$$


The sign of the function $f\left(\hat{d}_{i}(t)-\hat{d}_{j}(t)\right)$ is positive, which means that the action probability $p_{j}(t)$ is decreased proportional to its current value, $p_{j}(t)$.

The probability action $p_{i}(t)$ is increased or decreased to ensure that the sum of all the action probabilities is unity. When all the reward estimates are higher than the reward estimate of action $\alpha_{i}$, the automaton increases all the probabilities $p_{j}(t+1)$. To ensure that the amount that these probabilities increase does not surpass the value of $p_{i}(t)$, Thathachar and Sastry introduced the term $\frac{p_{i}(t)}{r-1}$ in the updating equations.

There are two main differences between the Pursuit Algorithm and the TSE Algorithm. First, they differ in the method of deciding which probability actions are increased and which are decreased. The Pursuit Algorithm increases only the probability of the action corresponding to the highest estimate, whereas the TSE Algorithm increases the probabilities for all the actions with a higher reward estimate that the estimate of the chosen action. Second, their updating equations differ. In increasing or decreasing a probability, the TSE Algorithm considers also the distance between estimates, incorporated in the term $f\left(\hat{d}_{i}(t)-\hat{d}_{j}(t)\right)$, whereas the Pursuit Algorithm takes into account only the distance between the probability at time $t$ and the probability that it aims for each action, 0 or 1 .

This algorithm has been shown to be $\varepsilon$-optimal [12, 14]. Also, Thathachar and Sastry presented simulation results to compare it with the $L_{R I}$ scheme. They have shown that for the same level of accuracy, the TSE Algorithm often converges at least seven times faster than the $\mathrm{L}_{\mathrm{RI}}$ scheme.

\section{Discrete Estimator Algorithms}

The Discrete Estimator Algorithms (DEA) were introduced as an approach to create even faster converging learning algorithms [5]. They emerged from applying the discretization "philosophy" to the existing estimator algorithms. In this way, the action probabilities are allowed to take a finite set of values and they use the reward estimates in their updating rules.

Lanctôt and Oommen have defined a set of properties that every "good" discrete estimator algorithm must posses [5]. These properties are known as the Property of Moderation and the Monotone Property.

Property 1: A DEA with $r$ action and a resolution parameter $N$ is said to posses the property of moderation if the maximum magnitude by which an action probability can decrease per iteration is bounded by $\frac{1}{r N}$.

The monotone property can be stated as below:

Property 2: Suppose there exists an index $m$ and a time instant $t_{0}<\infty$, such that $\hat{d}_{m}(t)>\hat{d}_{j}(t)$ for all $j \neq m$ and all $t \geq t_{0}$. A DEA is said to posses the Monotone Property if there exists an integer $N_{0}$ such that for all resolution parameters $N>N_{0}, p_{m}(t) \rightarrow 1$ with probability one as $t \rightarrow \infty$.

This means that if the estimate of reward of an action $m$ remains the maximum estimate after a certain point in time, then a DEA has the monotone property if it steadily increases the probability of choosing $\alpha_{m}$ to unity.

These properties are necessary in proving that a DEA is $\varepsilon$-optimal. Lanctôt and Oommen have proved that any discretized estimator algorithm possessing both of these properties is $\varepsilon$-optimal [5].

The discrete versions of $a$ Pursuit Algorithm and the TSE Algorithm are presented in the next subsections.

\subsection{The Discrete Pursuit Algorithm}

Lanctôt and Oommen introduced a discretized version of the Pursuit Algorithm [4] based on the rewardinaction learning "philosophy", denoted by DP RI. The differences between the discrete and continuous version of the Pursuit algorithms occur only in the updating rules for the action probabilities. The discrete Pursuit algorithm makes changes to the probability vector $\mathbf{P}(t)$ in discrete steps, whereas the continuous version uses a continuous function to update $\mathbf{P}(t)$. Being a rewardinaction algorithm, the action probability vector $\mathbf{P}(t)$ is updated only when the current chosen action is rewarded. If the current action is penalized, the action probability vector $\mathrm{P}(t)$ remains unchanged, which implies that the algorithm uses the estimates in updating the action probability vector $\mathbf{P}(t)$ only if the Environment rewards the chosen action. When the chosen action is rewarded, the algorithm decreases the probability for all the actions that do not correspond to the highest estimate, by a step $\Delta$, where $\Delta=\frac{1}{r N}$. In order to keep the sum of the components of the vector $\mathbf{P}(t)$ equal to unity, the $\mathrm{DP}_{\mathrm{RI}}$ increases the probability of the action with the highest estimate by an integral multiple of the smallest step size $\Delta$.

Oommen and Lanctôt proved that the $\mathrm{DP}_{\mathrm{RI}}$ has the following property.

Theorem VII: The DPRI satisfies both the properties of moderation and monotonicity, and is $\varepsilon$-optimal in all stationary random Environments.

Proof: The proof of the result is due to Oommen and Lanctôt and is given in $[2,4,5]$.

Oommen and Lanctôt performed simulations of the $\mathrm{DP}_{\mathrm{RI}}$ in some benchmark Environments and the results have been compared against the results of the $\mathrm{CP}_{\mathrm{RP}}$ algorithm. The results have shown that in some difficult Environments, the DP $\mathrm{RI}_{\text {I }}$ requires only $50 \%$ of the number of iterations required for its continuous version. In a ten-action Environment, the $\mathrm{DP}_{\mathrm{RI}}$ algorithm required $69 \%$ of the iterations required by the $\mathrm{CP}_{\mathrm{RP}}[4,5]$.

A description of the algorithm is given below. 


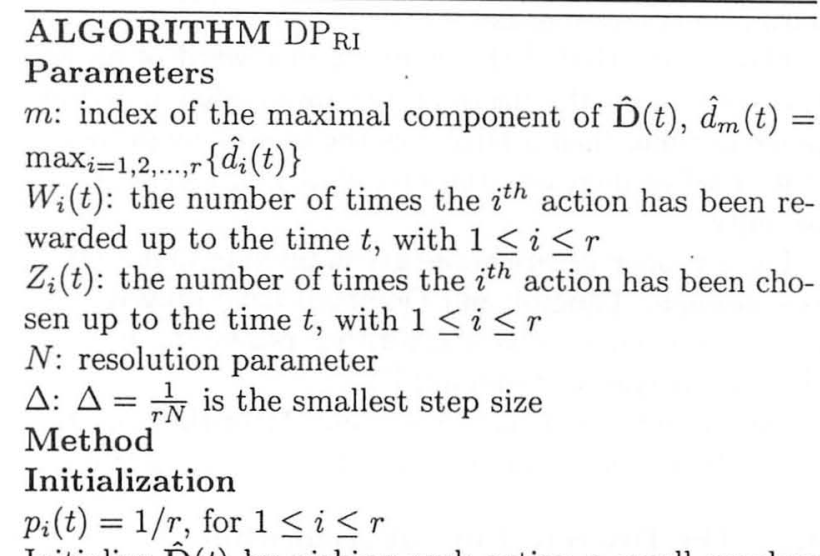

Initialize $\hat{\mathbf{D}}(t)$ by picking each action a small number of times

\section{Repeat}

Step 1: At time $t$ pick $\alpha(t)$ according to probability distribution $\mathbf{P}(t)$. Let $\alpha(t)=\alpha_{i}$.

Step 2: Update $\mathbf{P}(t)$ according to the following equations:

For all $j \neq m$,

If $\beta(t)=0$ and $p_{m}(t) \neq 1$

$$
\begin{aligned}
p_{j}(t+1) & =\max \left\{p_{j}(t)-\Delta, 0\right\} \\
p_{m}(t+1) & =1-\sum_{\forall j \neq m} p_{j}(t+1)
\end{aligned}
$$

Else

$$
p_{j}(t+1)=p_{j}(t) \text { for all } 1 \leq j \leq r
$$

Step 3: Update $\hat{\mathbf{D}}(t)$ as done in Algorithm $\mathrm{CP}_{\mathrm{RP}}$ End Repeat END ALGORITHM DP $P_{R I}$

\subsection{The Discrete TSE Algorithm}

Oommen and Lanctôt also presented the discretized version of the TSE Algorithm, denoted by DTSE [5]. As the authors have said, "the design of this algorithm is merely a compromise between the necessity of having the algorithm posses the moderation and monotone properties while possessing as many qualities of the continuous algorithm as possible" [5].

Oommen and Lanctôt have justified the transformation of the TSE Algorithm into a discretized one, by analyzing each factor that is part of the updating rules of the TSE Algorithm. The parameter $\lambda$, representing the maximum that the continuous probability component can change, has been replaced by the integer $\theta$. The term $f\left(\hat{d}_{i}(t)-\hat{d}_{j}(t)\right)$ was preserved in the DTSE Algorithm, representing a factor of the difference between the reward estimates. The term $\left(S_{i j}(t) p_{i}(t)+S_{i j}(t) \cdot \frac{p_{i}(t)}{r-1} \cdot\left(1-p_{j}(t)\right)\right)$ has been transformed to $\left(S_{i j}(t)+S_{j i}(t) \cdot \frac{1}{r-1}\right)$ by eliminating the continuous dependency on the probability vector, as part of the discretization process.

The resultant factor $\theta \cdot f\left(\hat{d}_{i}(t)-\hat{d}_{j}(t)\right) \cdot\left(S_{i j}(t)+S_{j i}(t)\right.$. $\frac{1}{r-1}$ ) determines the maximum value that a probability will be increased or decreased. In order to make these changes discrete, the above factor has to be represented in terms of the number of $\Delta$ - steps that preserve also the probabilities in the interval $[0,1]$. To do this, two new functions have been introduced: $\operatorname{Rnd}(x)$ and $\operatorname{Check}\left(p_{i}(t), p_{j}(t), x\right)$. The $\mathrm{Rnd}()$ function rounds up its parameter so that its value is always an integer. The Check() function calculates the largest integer multiple of $\Delta$, between 1 and $x$ that can be added to $p_{i}(t)$ and subtracted from $p_{j}(t)$, and which simultaneously preserves these probabilities in the interval $[0,1]$.

The algorithm modifies first the value of the action probability that has the highest reward estimate to guarantee that this value will always increase. A description of the algorithm is given below.

\section{ALGORITHM DTSE}

\section{Parameters}

- $m, W_{i}(t), Z_{i}(t), S_{i j}(t)$ are the same as in the TSE Algorithm

$\Delta=1 / r N \theta$, with $\theta$ (an integer) being the maximus any component can change by.

$\operatorname{Rnd}(x)$ rounds up $x$ to one of $\{-\theta,-\theta+1,-\theta+$ $2, \cdots, \theta-1, \theta\}$.

Check $\left(p_{i}(t), p_{j}(t), x\right)$ returns the largest integer $w \leq x$ such that $0 \leq p_{i}(t)+w \Delta, p_{j}(t)-w \Delta \leq 1$.

\section{Method}

Initialization

$p_{i}(t)=1 / r$, for $1 \leq i \leq r$

Initialize $\hat{\mathbf{D}}(t)$ by picking each action a small number of times

Repeat

Step 1: At time $t$ pick $\alpha(t)$ according to probability distribution $\mathbf{P}(t)$; Let $\alpha(t)=\alpha_{i}$

Step 2: Update $\mathbf{P}(t)$ according to the following equations:

For each action $j$, starting with $m$ Do

$$
\begin{aligned}
\text { change } & =\operatorname{Rnd}\left(\theta \cdot f\left(\hat{d}_{i}(t)-\hat{d}_{j}(t)\right) \cdot S\right) \\
p_{j}(t+1) & =p_{j}(t)-\Delta \cdot \operatorname{Check}\left(p_{i}(t), p_{j}(t), \text { change }\right) \\
p_{i}(t+1) & =p_{i}(t)+\Delta \cdot \operatorname{Check}\left(p_{i}(t), p_{j}(t), \text { change }\right) \\
\text { where } S & =S_{i j}(t)+S_{j i}(t) \cdot \frac{1}{r-1}
\end{aligned}
$$$$
\text { EndFor }
$$

Step 3: Update $\hat{\mathbf{D}}(t)$ as done in Algorithm $\mathrm{CP}_{\mathrm{RP}}$.

End Repeat

\section{END ALGORITHM DTSE}

Oommen and Lanctôt proved that the DTSE has the following property.

Theorem VIII: The DTSE algorithm satisfies both the properties of moderation and monotonicity, and is E-optimal in all stationary random Environments.

Proof: The proof of the result, due to Oommen and Lanctôt, can be found in $[2,4,5]$. 
The authors have also performed simulations in order to study the performance of the DTSE in comparison to its continuous version. Some of the results are presented in the following table:

Table 3: The number of iterations until convergence in two-action Environments for the Continuous and Discretized TSE Algorithms [5].

\begin{tabular}{|c|c|c|c|}
\hline \multicolumn{2}{|c|}{ Probability of Reward } & \multicolumn{2}{c|}{ Time for Convergence } \\
\hline Action 1 & Action 2 & Continuous & Discrete \\
\hline 0.800 & 0.200 & 28.8 & 24.0 \\
\hline 0.800 & 0.400 & 37.0 & 29.0 \\
\hline 0.800 & 0.600 & 115.0 & 76.0 \\
\hline 0.800 & 0.700 & 400.0 & 380.0 \\
\hline 0.800 & 0.750 & 2200.0 & 1200.0 \\
\hline 0.800 & 0.775 & 8500.0 & 5600.0 \\
\hline
\end{tabular}

In their simulation, the algorithms were required to reach a standard rate of accuracy of making no errors in convergence in 100 experiments. The initialization of the reward estimate vector was done in 20 iterations, which are included in the results shown in Table 3. The results have shown that the continuous TSE algorithm was from 4 to $50 \%$ slower that the discrete TSE. For example, with $d_{1}=0.8$ and $d_{2}=0.775$, the TSE algorithm took an average of 8,500 iterations to converge and the DTSE required only 5,600 .

Some comparisons were also made between all the estimator algorithms, in two benchmark ten-action Environments.

Table 4: Comparison of the discrete and continuous estimator algorithms in benchmark ten-action Environments [5].

\begin{tabular}{|c|c|c|c|}
\hline Environment & Algorithm & Continuous & Discrete \\
\hline$E_{A}$ & Pursuit & 1140 & 799 \\
\hline$E_{A}$ & TSE & 310 & 207 \\
\hline$E_{B}$ & Pursuit & 2570 & 1770 \\
\hline$E_{B}$ & TSE & 583 & 563 \\
\hline
\end{tabular}

Note: The reward probabilities of the Environments are: $E_{A}: 0.70,0.50,0.30,0.20,0.40,0.50,0.40,0.30,0.50,0.20$ $E_{B}: 0.10,0.45,0.84,0.76,0.20,0.40,0.60,0.70,0.50,0.30$

These Environments were the same ones used to compare the continuous estimator algorithms to the $\mathrm{L}_{\mathrm{RI}}$ scheme. The estimator algorithms sampled all $10 \mathrm{ac}-$ tions, 10 times each, to initialize the estimate vector. These 100 extra iterations are included in the results presented in Table 4. As the simulations of the twoaction estimator algorithms showed, the continuous version of the TSE algorithm is slower than the discrete version; for example, in the Environment referred as $\mathrm{E}_{\mathrm{A}}$, the DTSE takes 207 iterations to reach the endstate and the TSE takes 310 .

These results also show that the TSE algorithm is faster than the Pursuit Algorithm. In the same Environment, the continuous Pursuit Algorithm required 1,140 iterations to converge and the TSE algorithm re- quired only 310 . The same observation applies to their discrete versions.

\section{Conclusions}

This paper deals with the theory of stochastic Learning Automata (LA) which are probabilistic finite state machines that have been used to model how biological systems can learn. In particular, it concerns the family of Variable Structure Stochastic Automata (VSSA) which are LA implemented by using action probability updating rules. These rules may or may not depend on estimates from the Environment being investigated. The initial LA utilized updating rules which worked with the continuous probability space. In this talk, we have described how LA can also be designed by discretizing the probability space. The paper detailed the design and analysis of both continuous and discretized LA, and highlighted the subtle differences between the corresponding learning machines, their convergence properties, and their learning capabilities. Table 5 summarizes the convergence characteristics of these LA in their continuous and discretized forms.

Table 5: Comparison between continuous and discrete linear VSSA.

\begin{tabular}{|c|c|c|c|}
\hline LA & Type & Markov Nature & Convergence \\
\hline $\mathrm{L}_{\mathrm{RI}}$ & Continuous & Absorbing & Always $\varepsilon$-optimal \\
\hline $\mathrm{DL}_{\mathrm{RI}}$ & Discrete & Absorbing & Always $\varepsilon$-optimal \\
\hline $\mathrm{L}_{\mathrm{IP}}$ & Continuous & Ergodic & Always Expedient \\
\hline $\mathrm{DL}_{\mathrm{IP}}$ & Discrete & Ergodic & Always Expedient \\
\hline $\mathrm{ADL}_{\mathrm{IP}}$ & Discrete & Absorbing & Always $\varepsilon$-optimal \\
\hline $\mathrm{L}_{\mathrm{RP}}$ & Continuous & Ergodic & Always Expedient \\
\hline $\mathrm{DL}_{\mathrm{RP}}$ & Discrete & Ergodic & $\begin{array}{l}\text { Only } \varepsilon \text {-optimal } \\
\text { if }\left(c_{m}<0.5\right)\end{array}$ \\
\hline $\mathrm{ADL}_{\mathrm{RP}}$ & Discrete & Absorbing & Always $\varepsilon$-optimal \\
\hline $\mathrm{MDL}_{\mathrm{RP}}$ & Discrete & Ergodic & Always $\varepsilon$-optimal \\
\hline $\mathrm{P}_{\mathrm{RI}}$ & Continuous & Pursuit-based & Always $\varepsilon$-optimal \\
\hline$P_{R P}$ & Continuous & Pursuit-based & Always $\varepsilon$-optimal \\
\hline $\mathrm{DP}_{\mathrm{RI}}$ & Discrete & Pursuit-based & Always $\varepsilon$-optimal \\
\hline $\mathrm{DP}_{\mathrm{RP}}$ & Discrete & Pursuit-based & Always $\varepsilon$-optimal \\
\hline TSE & Continuous & Estimator-based & Always $\varepsilon$-optimal \\
\hline DTSE & Discrete & Estimator-based & Always $\varepsilon$-optimal \\
\hline
\end{tabular}

\section{Acknowledgements}

The author is grateful to the various colleagues and students he has worked with in the area of Learning Automata. In particular he thanks Professor Thathachar, to whom this paper is dedicated. He also records his gratitude to his co-authors, Dr. Hansen and Christensen, and his previous students M. Agache, D. Calitoiu, K. Lanctot and S. Misra, among others. Various portions of this paper are taken from documents that were prepared with these students, and he acknowledges their help in this regard. He is also thankful to Q. $\mathrm{Ke}$ and $\mathrm{C}$. Astudillo who helped with the preparation of the final document. 
The author is especially grateful to Professor Baba for his friendship and kindness with regard to the SSS'08 conference in Japan in 2008.

\section{References}

[1] M. Agache, Estimator Based Learning Algorithms. M.C.S. Thesis, School of Computer Science, Carleton University, Ottawa, Ontario, Canada, 2000.

[2] M. Agache and B. J. Oommen, "Generalized pursuit learning schemes: New families of continuous and discretized learning Automata", IEEE Transactions on Systems, Man, and Cybernetics, Part B, Vol. 32, No. 6, 2002, pp. 738-749.

[3] S. Lakshmivarahan, Learning Algorithms Theory and Applications. Springer-Verlag, 1981.

[4] J.K. Lanctôt, Discrete Estimator Algorithms: A Mathematical Model of Computer Learning. M.Sc. Thesis, Dept. Math. Statistics, Carleton Univ., Ottawa, Canada, 1989.

[5] J. K. Lanctôt and B. J. Oommen, "Discretized estimator learning automata", IEEE Transactions on Systems, Man, and Cybernetics, SMC-22(6):14731483, November/December 1992.

[6] K. Najim and A. S. Poznyak, Learning Automata: Theory and Applications. Pergamon Press, Oxford, 1994.

[7] K. S. Narendra and M. A. L. Thathachar, Learning Automata: An Introduction. Prentice-Hall, New Jersey, 1989.

[8] M. S. Obaidat, G. I. Papadimitriou and A. S. Pomportsis, "Learning automata: Theory, paradigms, and applications", IEEE Transactions on Systems, Man, and Cybernetics, Part B, Vol. 32, December 2002, pp. 706-709.

[9] B. J. Oommen and J. P. R. Christensen, " $\epsilon$ optimal discretized reward-penalty learning automata", IEEE Transactions on Systems, Man, and Cybernetics, SMC-18, May/June 1988, pp. 451-457.

[10] B. J. Oommen and E. R. Hansen, "The asymptotic optimality of discretized linear reward-inaction learning automata", IEEE Transactions on Systems, Man, and Cybernetics, SMC-14, May/June 1984, pp. 542-545.

[11] B. J. Oommen, "Absorbing and ergodic discretized two action learning automata", IEEE Transactions on Systems, Man, and Cybernetics, Vol. 16, March/April 1986, pp. 282-293.
[12] P. S. Sastry, Systems of Learning Automata: Estimator Algorithms Applications. Ph.D. Thesis, Department of Electrical Engineering, Indian Institute of Science, Bangalore, India, June 1985.

[13] M. A. L. Thathachar and B. J. Oommen, "Discretized reward-inaction learning automata", Journal of Cybernetics and Information Science, Spring 1979, pp. 24-29.

[14] M. A. L. Thathachar and P. S. Sastry, "A new approach to designing reinforcement schemes for learning automata", IEEE Transactions on Systems, Man, and Cybernetics, SMC-15, 1985, pp. 168-175. 